\title{
Notas sobre la recepción de la literatura catalana moderna en Italia: el caso de Josep Pla
}

\author{
Notes about the reception of modern Catalan Literature \\ in Italy: the case of Josep Pla \\ LEONARDO VILEI (UCM) \\ lvilei@ucm.es \\ Recibido: abril de 2015. Aceptado: mayo de 2015
}

\begin{abstract}
Resumen: Josep Pla está considerado en Italia como uno de los escritores catalanes más importante del siglo XX. Sin embargo, existen muy pocas traducciones de su obra, la cual parece confinada a los estudios académicos o circunscrita al área geográfica de la isla de Cerdeña. La recepción italiana de Pla se inscribe solo parcialmente en el cuadro general de difusión de la literatura catalana, a su vez sugiere alguna peculiaridades.
\end{abstract}

Palabras clave: Josep Pla, literatura italiana, literatura catalana, literatura comparada, Cerdeña.

\begin{abstract}
Josep Pla is considered in Italy as one of the most important $20^{\text {th }}$ century Catalan writers. In spite of this, the translations from his books are few and his work is circumscribed to the academic studies or to the delimited area of Sardinia. Pla's reception is parcially related to the circulation of the whole modern Catalan literature in Italy.
\end{abstract}

Key words: Josep Pla, Italian studies, Catalan studies, comparative studies, Sardinia.

El Grand Tour, ese viaje a Italia emprendido con propósitos formativos por pintores, literatos y demás apasionados del arte y de la historia, estimuló, a caballo entre los siglos XVII y XVIII, una vigorosa producción de escritos y pinturas que contribuyeron a aumentar su atractivo. La mitificación de la península, de sus famosas ciudades e inolvidables paisajes se puso de manifiesto en un vasto repertorio de imágenes capaces por sí solas de avivar el deseo de compartir la experiencia en un cada vez más abundante número de curiosos y admiradores del país, entre los cuales resonaban con fuerza nombres tan ilustres como los de Montaigne, Goethe, Stendhal y Ruskin. Una tradición tal planteaba 
necesariamente la confrontación de la Italia real con las expectativas generadas por las descripciones más sugerentes y famosas. Fue el genio de Marcel Proust quien con su perspicaz descripción de las tensiones intelectuales y sentimentales que preceden al soñado viaje a Venecia ${ }^{1}$, precisó de forma magistral ese conflicto entre la sobreabundancia de expectativas y la evidencia de la experiencia real.

Josep Pla se sitúa en esta ilustre tradición de viajeros y escritores, a raíz de las numerosas temporadas pasadas en el Bell País. Sus viajes, desde los primeros años veinte hasta bien entrados los sesenta, son la ocasión para que su atenta y sutil mirada perciba la dialéctica establecida entre las monumentales herencias de un imponente pasado y las contradictorias, exitosas o fracasadas, experiencias del presente.

Pero la cercanía de Pla con Italia no es producto exclusivo de sus viajes, sino que se sustenta además en un profundo conocimiento de la literatura italiana, con referencias constantes en su obra a autores como Leopardi, Dante, Manzoni o Carducci, y en un juicio nada convencional sobre la cultura italiana, tanto en el plano histórico como en el estético. Con estos antecedentes, teniendo en cuenta además las circunstancias políticas de los años veinte del siglo pasado, el ampurdanés estaba abocado casi inevitablemente a dar forma a un diálogo entre Italia y Cataluña, dos países que, en las palabras de Rossend Arqués, pone enfrente el uno al otro de manera especular:

[...] nostre escriptor es reflecteix en el mirall d'aquesta terra i compara la imatge real o «mental» que se n'ha fet amb la realitat més real i física que mental de la cultura de la qual prové; llocs que es disposaran en l'obra i en la ment de l'escriptor en l'espai antagònic que va del valor positiu gairebé absolut (Itàlia) al valor negatiu no pas del tot absolut però poc li falta (el País del qual prové). (Rossend 2002: 188.)

El amor por Italia y el entendimiento de su complejidad sustentan las numerosas páginas que, en el conjunto de su enorme producción, dedica a este país; un amor que, sin embargo, no ha encontrado en la cultura italiana la reciprocidad que cabría esperar. Pla en el Bel Paese es un escritor sólo parcialmente traducido y poco conocido si exceptuamos los ámbitos especializados dedicados a las letras catalanas. Nos ocuparemos pues en este artículo de la recepción del ampurdanés en la cultura italiana, teniendo en cuenta los hechos disponibles y tratando de comprenderlos conforme a las distintas etapas históricas y culturales en que aquellos tienen lugar.

1 Desde las primeras páginas de Du côté de chez Swann, el personaje de la Recherche relata como la primera idea de Venecia surge en él a partir de un dibujo de Tiziano que tiene como fondo la laguna. Las referencias a la ciudad son múltiples a lo largo de toda la obra, con una especial influencias de Stones of Venice de Ruskin, hasta que la realidad del viaje se concretiza con todas sus consecuencias, entre las cuales encontramos una larga reflexión acerca de la distancia entre la ciudad imaginada y la ciudad conocida. Para profundizar se remite a Bettini, S. (2006) Venezia. Nascita di una città, Milano, Neri Pozza. 


\section{LA LITERATURA CATALANA EN ITALIA: UN CUADRO GENERAL}

En primer lugar parece oportuno fijar el cuadro en que se inserta la recepción de Pla, enfocando esa delimitación desde la perspectiva del conocimiento y de la sensibilidad italiana respecto a la literatura y cultura catalana.

El primer texto dedicado a la literatura catalana en el Regno d'Italia se publica en 1878. Se trata de un ensayo del estudioso Enrico Cardona en el que el autor manifiesta una sensibilidad de tipo risorgimentale ${ }^{2}$, sensibilidad esta bien comprensible vistos la fecha de la publicación y el clima que la acompaña. De Cataluña se subraya la laboriosidad de su gente, la fertilidad de sus tierras, la franqueza de su carácter; todo ello parece estar en implícita oposición a la «ociosa, polvorienta y orgullosa España ${ }^{3}$, por citar tres de los caracteres negativos que se asociaban a una nación considerada entonces en el ocaso de su antiguo esplendor. En la misma línea, una nota final de Schlegel recuerda la grandeza de la literatura española entre los siglos XV y XVII, lo que le lleva a «deplorare davvero la floridezza e la prosperità perdute da un nobile e valoroso paese [...]» (Cardona 1878: 129).

La especificidad de la literatura catalana se determina a partir de estas premisas, rescatándola de su histórico aletargamiento y de la confusión con su hermana castellana: «una Storia tutta sua del pensiero, una Letteratura propria, ed una lingua peculiare [...]» (Cardona 1878: 6). Las referencias a los más recientes estudios de la época sobre la lengua catalana, procedentes en gran parte de Francia y Alemania, países auctoritas por antonomasia en Italia, fundamentan el trabajo de Cardona, el cual se introduce con una breve historia de la península ibérica para luego abordar la formación de sus lenguas, destacando la influencia del modo provenzal en la aparición de las primeras experiencias literarias. La amplia reseña dedicada a la época medieval catalana y el análisis del esplendor de su literatura en ese periodo, hasta la pérdida de la autonomía política y consecuente decadencia de sus manifestaciones culturales, terminan con el augurio de que futuros estudiosos, apasionados por el tema, aportarán su contribución para que en Italia se de a conocer mejor una «Letteratura non troppo nota» (Ivi: 125), y ello en aras

2 Risorgimento es la palabra que se emplea en Italia para la unificación del País, el proceso histórico que a lo largo del siglo XIX llevó a la unión de los diversos estados en que estaba dividida. A la unificación territorial y administrativa siguió la unificación cultural y lingüística, proceso dentro del cual el canon literario jugó un papel determinante, en cuanto precursor de los ideales patrióticos y políticos. El Romanticismo, de hecho, tuvo en Italia una declinación de tipo nacional y en este sentido se establece una cercanía con Cataluña.

${ }^{3} \mathrm{La}$ «leyenda negra» de la opresión española en su dominación italiana, considerada durante el Risorgimento como la peor de entre las dominaciones extranjeras, se reflejaba también en personajes literarios, como es el Don Rodrigo de Manzoni en I Promessi sposi; el caballero español despótico, parásito y rufián, es un perfecto ejemplar de un tipo que posteriormente se identificó con todo el sur de Italia. Hasta por lo menos 1925, año de la publicación de Storia del Regno di Napoli de Benedetto Croce (Roma-Bari, Laterza), el supuesto atraso del Meridione italiano confirmaba la supuesta pésima herencia de lo español. Croce inauguró un debate historiográfico vigente todavía, que restituye a la Italia pre-unitaria, y por ende a los gobiernos de los diferentes reinos y estados que la componían, una visión menos retórica y más matizada. 
tanto de la propia especificidad del tema, como de su encaje en una más compleja aproximación a las lenguas románicas.

En el ensayo que estamos comentando se constata una oposición entre el pasado positivo y el presente negativo de España, oposición que parece incentivar la diferenciación de un específico ámbito catalán. No podía ser de otra forma, desde luego: Italia misma acababa de nacer como un nuevo estado nacional que consideraba su tradición literaria como núcleo determinante para la formación de su identidad como tal (Raimondi 1997: passim), identidad fragmentada hasta aquel momento por el poder extranjero que, en el caso del Regno delle due Sicilie, era precisamente el poder borbónico español. Si bien la historiografía sobre el Risorgimento italiano ha corregido de forma notoria las deformaciones de una visión enfáticamente nacionalista — la cual se construyó alrededor de tres cuestiones principales: la tiranía del Imperio Austro-húngaro, el atraso y el parasitismo borbónicos, y la opresión antimoderna del Estado Pontificioes cierto que durante mucho tiempo la visión negativa de lo español, cuyo siglo XIX fue testigo de tres guerras civiles, impregnó la mentalidad de la nueva Italia, que eligió inspirarse, por distintas razones, en Francia, Inglaterra y Alemania. Es suficiente, al respecto, citar el íncipit del estudio de Cardona: «Quella Spagna che oggi è tanto rinomata per le sue lunghe e crudeli guerre civili, le quali non hanno saputo da ultimo che in tutti i modi impoverirla, fu pure in gran fama una volta per la sua potenza politica, nei giorni della monarchia fondata da Carlo V, e pel suo svolgimento intellettuale [...]» (Cardona 1878: 5). Con estas premisas, la simpatía hacia la cuestión catalana era inevitable.

Se nota sin embargo en el estudio de Cardona, que persigue con elocuente afán dar a conocer la cultura catalana, la ausencia de cualquier referencia a la Renaixença, la cual sí se incluirá más tarde en el grupo de los fenómenos decimonónicos románticos ligados a la construcción de una identidad nacional alrededor de una recobrada identidad lingüística y literaria. Es necesario esperar al nuevo siglo para que los hispanistas italianos recojan esta correspondencia entre Italia y Cataluña, que se confirmará más sosegadamente en la sucesiva dialéctica Novecentismo/Noucentisme. Esta correspondencia se adelanta ya en un libro que recoge los coloquios del artista argentino José León Pagano con varios autores catalanes, publicado en Italia en 1902 y que dio a conocer, en el mundo literario italiano, la existencia de una generación de escritores catalanes que reclamaba su lugar en las letras europeas de la época. A este respecto - Pagano se encontraba en Italia para perfeccionarse como artista y entró en contacto con escritores y pintores - sabemos que el argentino, después de la publicación de su libro, recibió el elogio de Gabriele D'Annunzio: «Sin pizca de vanidad, cito un juicio de D'Annunzio respecto al primer tomo dedicado a la literatura catalana. Por lo novísimo de esa materia en Italia, el poeta del Fuego me llamó «rivelatore dell' ardente e combattente Catalogna»» (Pagano 1964: 27).

En el ámbito académico es preciso destacar los estudios de Mario Casella, dantista, filólogo y gran conocedor de la compleja historia lingüística y literaria de la península ibérica. En 1918 publicó Agli albori del Romanticismo e del moderno Rinascimento catalano, un estudio que vino a enmendar la escasez de noticias que circulaban en Italia sobre la Renaixença y que durante mucho tiem- 
po representó una referencia para los estudiosos del tema. Casella establecía la correspondencia de la Renaixença con el Risorgimento en un plano no solo cultural, bajo la común declinación nacionalista del romanticismo, sino también histórico, con la presencia de emigrados liberales italianos en la redacción de la revista «El Europeo» ${ }^{4}$ :

Nell'Europeo dobbiamo trovare la prima causa dell'influenza italiana in Catalogna durante la prima metà del secolo passato, dell'interesse che per i nostri scrittori, e fra tutti il Manzoni ${ }^{5}$, serbarono i primi fattori del movimento regionalista, il Quadrado, il Llausás, i due fratelli Milá y Fontanals. Divulgatore dei nostri e imbevuto delle loro dottrine si mostra l'Aribau. (Casella 1966: 336.)

En el camino de los parecidos y de las influencias, Casella ve la sombra de Manzoni - Addio ai monti, la célebre evocación de las montañas del lago de Como que hace Lucia, personaje principal femenino de I promessi sposi-en una estrofa de Oda a la patria catalana de Aribau -A Déu siau, turóns, per sempre á Déu siau-. Destaca también Casella las traducciones al catalán de algunos autores italianos, publicadas en el diario «El Vapor» ${ }^{6}$, como es el caso de la Noya Fugitiva de Tommaso Grossi ${ }^{7}$ (Casella 1966: 344-347). Gracias a estas revistas, en las cuales el templado romanticismo lombardo ejerció su influencia en tierra catalana, se prepara, según expone el autor, el renacer de un «neo-provenzalismo» y un «catalanesimo», surgidos como «reazione a quello spirito di uniformità», para lograr una mayor «comprensione delle realtà tradizionali di natura e di storia e a un riconoscimento più chiaro dell'individualità regionale nell'unità superiore dello Stato» (Casella 1966: 351). Cataluña, lejos de estar volcada en una visión tradicionalista de sí misma, destaca por su voluntad industrial, su vocación cosmopolita, la vitalidad de su comercio y el pragmatismo de su organización:

Posta all'avanguardia della civiltà spagnola e schiusa alle correnti intellettuali d'Europa, rinnovate nel fecondo lavoro le sue energie e riannodata l'interrotta tradi-

${ }^{4}$ «El Europeo» fue una revista aparecida en Barcelona entre los años 1823 y 1824, importante por ser uno de los elementos introductores del Romanticismo en España. Sus editores fueron los emigrados liberales italianos Fiorenzo Galli y Luigi Monteggia, los catalanes Buenaventura Carles Aribau y Ramón López Soler y el inglés de origen alemán Carlos Ernest Cook. Casella establece una relación entre la revista e «Il Conciliatore», la publicación más representativa del romanticismo italiano.

5 Acerca de la recepción de Alessandro Manzoni en Cataluña, se destaca, a distancia de casi cien años de su fortuna en «El Europeo», un admirado retrato del escritor lombardo en las páginas de Cartes d'Itália dedicadas a la ciudad de Milán. Cfr. Milá, en Pla 1983: 213-220.

6 «El Vapor» fue un periódico editado en Barcelona entre los años 1833 y 1837. De tendencia liberal, tuvo gran acogida entre la población. Su contenido era político, mercantil y literario. El 24 de agosto de 1833, publicó el poema Oda a la Patria de Bonaventura Carles Aribau.

7 Es interesante leer la nota que acompañaba la traducción de La fuggitiva, que por otro lado fue escrita en dialecto milanés, para entender el parecido posible entre la cultura lombarda, propulsora en Italia del romanticismo literario con mayor repercusión, y el romanticismo catalano: «[Grossi es] uno de los que más honran esa literatura lombarda que tantas hojas añade al clásico laurel de Italia» (cit. en Casella 1966: 347). 
zione storica, Catalogna, che da secoli era rimasta muta nella produzione letteraria della penisola, potè per bocca de' suoi filosofi, de' suoi economisti, de' suoi giureconsulti e de' suoi poeti dire in molte cose la prima parola [...]. (Ivi: 353 .)

\section{LA PRIMERA TRADUCCIÓN DE PLA AL ITALIANO: PIETRO BRINCS UOMO DI BAGUR}

Los frutos de los trabajos comentados se aprecian en la primera Antologia di novelle catalane, aparecida en 1926, que presenta un canon de la moderna literatura catalana. La obra, editada por Giuseppe Ravegnani ${ }^{8}$, traduce los relatos de dieciséis escritores: Narcís Oller, Raimond Casellas, Santiago Rusiñol, Joan Maragall, Joaquim Ruyra, Victor Català, Prudenci Bertrana, Roig i Raventós, Gabriel Alomar, Alfons Maseras, Josep Carner, Alexandre Plana, Carles Soldevila, E. Duran-Reynals, Josep Pla y E. M. Ferrando. En la introducción de este volumen, Ravegnani anuncia una segunda serie con otros tantos autores ${ }^{9}$ «[...] se fortuna pari alla buona accoglienza avrà questo nostro disinteressato lavoro [...]» (Ravegnani 1926: 19), que, sin embargo, nunca se llegó a publicar, aunque desconocemos si ello se debió al escaso éxito de la primera o a otras razones.

La propuesta editorial merece ser tenida en cuenta no solo porque en ella encontramos la primera presentación de nuestro escritor al público italiano, sino porque representa un caso interesante de como la recepción de un texto por parte de la industria editorial va adaptándose al gusto y a la comprensión del público que lo recibe. A este respecto, se ha de destacar la elección de la palabra novella ${ }^{10}$ en el título del volumen, género este renovado en la tradición italiana a partir del siglo XIX, y elegido por maestros como Giovanni Verga, Gabriele D’Annunzio o Luigi Pirandello.

Para promover una cultura literaria que se considera importante, aunque poco conocida, el volumen incluye una amplia introducción a las letras catalanas, así como notas abundantes y perfiles biográficos de los autores. De este modo, la introducción opera como una presentación de la «Rinascenza della prosa catala-

8 Giuseppe Ravegnani (1895-1964) fue un conocido escritor, periodista, crítico literario, poeta y traductor.

9 Emili Vilanova, Gabriel Maura, Pere Corominas, Joaquim Folguera, Carles Riba, Ventura Gassol, Ramon Raventòs, M. S. Oliver, Agustì Calvet, Llorenç Riber, J. M. López-Picó, Pelay Briz, Josep Moratò, Pin y Soler, Pous i Pagés, Conrat Rure, E. D’Ors, F. Pujols, Ixart, Robert Robert.

${ }^{10}$ El género de la novella en el siglo XIX en Italia renueva la herencia de Boccaccio y su posterior fortuna durante el Rinascimento; entabla un diálogo con el relato romántico-social inglés (Dickens), la gran tradición del realismo ruso (Puskin, Gogol, etc.) y sobre todo el ejemplo del naturalismo francés, que impulsa la novellistica italiana en la dirección de la tranche de vie. Vita nei campi (1880) y Novelle rusticane (1883) de Verga, obras contemporáneas a los primeros relatos de Maupassant, marcan un camino en el que prima la ambientación rural, la vida campesina y pastoral, la honda conducta de los personajes. El término novella convive, a caballo entre el XIX y el XX, con muchas otras palabras que describen una narración breve — racconto, storiella, fantasia, bozzetto, scena, schizzo, racconto campestre - y sin embargo se impone por su difusión en la prensa y por su tradición en el cánon literario. Conforme va avanzando el nuevo siglo deja el paso al término racconto. 
na» esbozada a partir de «scintille, deboli e indecisi barbagli», por autores como Soler y Aribau, «assertori e propagatori della luce del Romanticismo europeo nella vecchia Spagna, sia castigliana che catalana» (Ravegnani 1926: 9).

La Renaixença se pone en relación con el Romanticismo europeo el cual ilumina la antigua tradición literaria catalana «ben viva sin dal secolo IX, ma però senza coscienza di sé medesima e senza veri e propri cultori». El autor subraya como aquella tradición, plenamente europea en su vitalidad medieval, no había muerto tras la decadencia del siglo XVI y se reavivaba ahora, no por casualidad, como experiencia europea de inspiración romántica y nacionalista, acorde con la visión del Risorgimento italiano, que veía justamente en la literatura el fundamento más vivo de una identidad nacional.

El juicio de Ravegnani acerca de la obra de los primeros románticos catalanes es más generoso al valorar su impulso patriótico que su calidad literaria; con respecto a los autores de la segunda mitad del siglo XIX sin embargo, el juicio cambia:

Con Verdaguer, Guimerà e Maragall a principio la nuova poesia catalana, non solo in quanto a bellezza lirica, ma anche come chiarificazione e assestamento di lessico, iniziandosi quell'opera di fissazione grammaticale e di ripulitura linguistica, che culminò poi nel filologo Pompeu Fabra [...]. (Ravegnani 1926: 13.)

Explicadas las bases de la Renaixença, el análisis de Ravegnani se dirige a la nueva prosa catalana del último cuarto del siglo XIX; según el autor, a partir de la obra de Emili Vilanova y de la de Narcis Oller, «il numero dei nuovi prosatori della piccola Catalogna divenne tale da uguagliare, se non superare, quello delle grandi nazioni letterarie: la Francia, la Germania, l'Italia» (Ivi: 14). El deseo de situar la prosa catalana en un cuadro europeo orienta la selección de los textos hacia el eje realismo-naturalismo, con una predilección por el drama rural, acentuando de esta manera el parecido con la tradición italiana del verismo.

La cercanía entre los cuentos catalanes y la novella italiana se fundamenta, además de en la recurrencia a la ambientación rural, en un mecanismo lingüístico parcialmente parecido. Giuseppe Ravegnani destaca aquellas expresiones que, tal como ocurre con la novella rusticana ${ }^{11}$ o barbarica $^{12}$, dan un color

11 Las Novelle rusticane (1883) di Giovanni Verga representan una de las obras clave del verismo, la declinación italiana del naturalismo, según la fórmula indicada por su principal teórico, el escritor Luigi Capuana. En ella encontramos la maduración de la estética verghiana, con la presencia de personajes de extracción social humilde, como pescadores, campesinos y pastores. Con respecto a Francia, es el campo y no la ciudad el centro de la atención del verismo, y por otra parte la psicología de los personajes no es mera consecuencia de un determinismo biológico. Fundamental, además, es la cuestión de la lengua; si bien los nuevos italianos iban aprendiendo un idioma antes desconocido o mal hablado por la mayoría, el dialecto mantuvo enteramente su predominio en el ámbito de las relaciones familiares, locales y personales. La necesidad de representar de forma verosímil esos ambientes, ponía a los escritores ante la cuestión de qué hacer con los dialectos, en muchos casos incomprensibles en otras regiones y provincias. Giovanni Verga es el que logra un milagro lingüístico, con una original mezcla del dialecto siciliano y otros registros y variantes del italiano.

${ }^{12}$ Con las Novelle della Pescara (1902) Gabriele D'Annunzio da prueba de su versatilidad literaria, dejando de lado los temas decadentistas y tomando como modelo al Verga siciliano. A la 
dialectal al estilo de algunos escritores, produciendo un tipo de regionalismo, o localismo, determinado por la mezcla de registros diferentes.

Dippiù, noi abbiamo osato e tentato di far sentire al lettore, con appropriate fedeltà, le diversità stilistiche dei singoli scrittori, per meglio valorizzarli nel loro completo valore e nelle loro peculiari virtù di grammatici. Qualsiasi attento lettore dovrebbe di questo accorgersi: e notare quale differenza esiste, per esempio, tra lo stile duro, angoloso, plastico del Pla e quello discorsivo, leggero, fragile dell'Oller. Così, abbiamo voluto aderire con la nostra traduzione a certo colorismo d'ambiente, prettamente catalano, ch'è nell'arte rurale della Català, del Ruyra, del Raventós, scrittori codesti che spesso si compiacciono di imbrillantare i loro periodi con espressioni locali, commerciali diremmo - per intenderci — dialettali, le quali, il più delle volte, sono introvabili anche nei vocabolari, maggiormente noti, del Vogel, del Bulbena y Tosell e del Rovira i Virgili. (Ravegnani 1926: 19.)

Josep Pla, del cual se recoge uno de sus relatos juveniles, destaca en la antología como uno de los escritores contemporáneos dignos de la mayor consideración. La novella traducida es Pietro Brincs, uomo di Bagur, que se había publicado en el número de enero de 1920 de la revista «D'acì d'allà» ${ }^{13}$, la cual se asocia a la revista italiana LLa lettura ${ }^{14}$ por temáticas y estilo. Acerca del escritor se subraya la «poverissima [...] bibliografia [...] sparsa nelle riviste catalane» (Ivi: 235); paupérrima por cantidad, pero no por calidad, pues «pur tuttavia, il Pla è uno dei più originali e forti prosatori della Catalogna moderna», afirmación a la que Ravegnani añade su cercanía con la mejor literatura rusa «per una certa rudezza stilistica e per la spiritualità dominante». Se trata de un juicio estético peculiar, que tal vez le hubiera intrigado al propio Pla, si pensamos que en su autorretrato en el Quadern Gris describe sus rasgos como procedentes del Cáucaso central e imagina una ascendencia de familia rusa, familia que en algún momento vino a parar en la «cloaca del Mediterráneo» ${ }^{15}$.

Hoy sabemos algo que era imposible conocer en 1926: el relato de Pla incluido en la antología no es especialmente representativo de su obra posterior. En él encontramos un personaje, un campesino cuyos gestos cotidianos están descritos

sensualidad mundana, romana y decadente, D'Annunzio opone aquí lo ancestral y pagano de su tierra, la región del Abruzzo, fuente de inspiración de su faceta bárbara y pánica.

13 «D'Ací i d'Allà» fue una revista cultural catalana que se editó en Barcelona entre 1918 y 1936, de aparición mensual hasta 1931 y trimestral desde entonces. Fue considerada la primera revista catalana de carácter europeo; su estilo fue ecléctico y disfrutó de una presentación cuidada y lujosa, gracias también a la colaboración con grandes fotógrafos.

14 «La Lettura» fue una revista mensual del diario «Corriere della Sera», pubblicata de 1901 a 1945. Su formato se inspiraba en los magazines ingleses, por la mezcla de ilustraciones, artículos y reportajes fotográficos. La crítica literaria y el enfoque de periodismo culto buscaban tanto el favor de los intelectuales, como de un amplio público burgués.

${ }_{15}$ Nos referimos al Autoretrat verídic promés a la senyora Lola S...i no enviat por excés del sentid del ridícul (Pla 1983: 150-159), donde leemos: «Tinc la cara notóriamente plana i els pòmuls amples i sortits. Això féu dir a alguns amics de Barcelona [...] que jo semblo un rus del Mediterrani». 
en tercera persona, y una voz diferente a la primera persona de su sucesiva escritura; del Pla posterior divisamos la minuciosidad descriptiva y la precisión lexical. Debido a lo reducido de su producción en aquel momento y quizás por la insatisfacción de poder decir tan poco acerca de un escritor que, Ravegnani intuye, presenta el potencial de un gran autor, el perfil biográfico que acompaña el relato es substituido por un autorretrato del propio Pla, parcialmente adaptado para la edición italiana: «Sento per la politica la noia dell'uomo che l'ha vissuta dappresso. Come uomo sensibile, sono nazionalista catalano e domando a tutti gli uomini sensibili d'Italia un po' di cordialità e di curiosità per il nostro problema» (Pla 1926: 236).

Si el relato elegido responde a la línea general que inspira el volumen - la novella de ambientación rural, el drama psicológico, el boceto de un mundo reducido - lo más representativo de la escritura de Pla se encuentra en el paratexto, sin que probablemente Ravegnani lo pudiese sospechar en aquella fecha. Si bien el crítico y estudioso demuestra una feliz intuición acerca del autorretrato del escritor ampurdanés, otorgándole, único caso en toda la antología, el espacio reservado al comentario biográfico, no se encuentra aún en la perspectiva necesaria para entender que aquello es precisamente el fragmento más representativo de la gran obra de Pla.

Mi chiamo Josep Pla i Casavadevall. Nacqui a Palafrugell, città industriale del basso Empordà, il giorno 8 del Marzo del 1897. Il mio nonno paterno era un piccolo proprietario rurale. La mia nonna pure era della campagna, ed un suo fratello, che studiava medicina a Barcellona, fu un acceso liberale e morì giovane, ucciso dalle disillusioni politiche. Il mio nonno paterno era uno scettico. [...]. (Pla 1926: 235.)

El Pla escritor de novelle rusticane debuta así delante del público italiano de forma en cierta medida equivocada con il senno di poi; después de este temprano comienzo, en el cual se le califica como el escritor más prometedor de su generación, sigue en Italia un larguísimo paréntesis de silencio hacia su obra, que ha durado hasta los años noventa.

\section{EL RETORNO DE CATALUÑA DESPUÉS DEL FRANQUISMO}

La interrupción de la difusión de la obra de Pla en Italia coincide solo en parte con un silencio sobre la literatura catalana, acerca de la cual, en efecto, hasta los años setenta no encontramos nuevos ensayos, antologías o estudios de la misma envergadura de los que se han citado anteriormente. Este corte puede explicarse por la situación política en España y su desconexión con el debate cultural europeo, después del catalizador simbólico que había representado la Guerra Civil. Sobre la España franquista, de todos modos, no habían caído ni el silencio, ni la desatención, aunque durante ese periodo la atención a lo catalán parece menguar, hasta que, en los años setenta, en el mundo académico nace la 
«Associazione italiana di studi catalani» ${ }^{16}$, creada en 1978 por profesores de filología, lingüística e historia. En este renovado interés hacia la singularidad de la literatura catalana en el contexto de la producción del área ibérica, la peculiaridad y la grandeza de Pla, cuya obra completa había mientras tanto emergido en España de forma ya casi definitiva, se pone de nuevo en evidencia; sin embargo no aparece ninguna nueva traducción de sus obras desde la ya lejana novella incluida en la Antologia de Ravegnani de 1926.

Analicemos al respecto ese renovado interés por la literatura catalana el ensayo de Giuseppe Grilli ${ }^{17}$, La letteratura catalana. La diversità culturale nella Spagna moderna, de 1979, donde el retorno de Cataluña en el horizonte cultural italiano aparece, mutate spoglie, en la crítica militante y con un enfoque decididamente politizado. El ensayo se abre con una profesión de fe política en defensa de la autonomía de la historia y literatura catalana; se apoya a continuación la aspiración independentista, o por lo menos una diferenciación de tipo nacional con respecto al resto de España. El autor, que fue lector en la Universidad de Barcelona entre 1969 y 1972, reconoce que su libro quiere promover el conocimiento y reconocimiento de una identidad nacional y cultural: «chi scrive è infatti in qualche modo catalanista» (Grilli 1979: 9), en sentido ideológico e histórico, y quiere difundir el conocimiento de las distintas facetas que configuran la idea de los Països Catalans. El análisis de Grilli comienza subrayando la diferencia catalana, no solo con respecto al resto de la península ibérica, sino también al conjunto de la romanitas. «La Catalogna esprime (...) tra il XIII e il XIV secolo, caratteri differenziali spiccati (...): lingua, folklore, diritto e letteratura hanno un' autonomia e già una tradizione» (Grilli 1979: 10).

Conforme a un enfoque de tipo político, tan abundante en la Italia de los años setenta, Grilli ofrece una explicación que puede ser adscripta al marco del materialismo-histórico, acerca de los fenómenos que se acumulan en la Cataluña en el siglo XX. Desde esta óptica el catalanismo

[...] ha così contribuito a mettere in luce le caratteristiche strutturali dello Stato spagnolo moderno, facendo rilevare la contraddizione oggettiva tra accentramento politico e realtà multinazionale [...]. Con lo sviluppo industriale e urbano del XX secolo si determina una situazione di immigrazione di grandi masse proletarie, e comunque ex contadine ed anche di ceto medio rurale e burocratico, maggioritariamente di altra nazionalità, ma con la stessa cittadinanza: l'uso che di questa mutazione demografica e culturale compie l'oligarchia dominante è di fondamentale importanza. (Grilli 1979: 12-13.)

$16 \mathrm{http}: / /$ www.aisc.cat/.

17 Actualmente Director del «Dipartimento di lingue, letterature e culture straniere», Università Roma Tre, Grilli se ha ocupado ampliamente de cultura catalana. Destacamos que en la Wikipedia existe una entrada sobre él exclusivamente en catalán, donde se dice: «Es llicencià en filologia el 1969, i romandrà com a lector a la Universitat de Barcelona fins a 1972. Des de 1983 és professor de Llengua i Literatura Catalana a l'Institut Universitari Oriental de Nàpols. Ha organitzat congressos dedicats a aspectes de la cultura catalana. Ha estat President d'Estudis Catalans a Itàlia, i de l'Associació d'Hispanistes Italians. Va rebre la Creu de Sant Jordi el 1996. El 2013 fou guardonat amb el Premi Sant Jordi de l'Institut d'Estudis Catalans». 
En conclusión, para Grilli el nacionalismo catalán se situa en el centro «di qualsiasi ipotesi di trasformazione anticapitalistica non solo dei paesi catalani, ma della Spagna tutta» (Ivi: 13), en cuanto puede obrar como un desencadente revolucionario, según la visión sugerida en la introducción a la edición italiana de los escritos de Marx sobre España, (Rubini, A. (1976) la Rivoluzione in Spagna, Firenze), a la cual el autor hace referencia directa.

El discurso del estudioso es acorde con una visión política reconocible en los paradigmas de una dialéctica de la historia de inspiración marxista, con fundamento en la cual explica la decadencia cultural de Cataluña, su posterior resurgimiento y la represión franquista que en esos momentos ya había terminado. Según Grilli, a la crisis de la lengua literaria en el siglo $\mathrm{XV}$, que se corresponde con una crisis institucional y con la ausencia de un centro impulsor de la hegemonía, sigue, a partir del siglo XVI, la fragmentación de la lengua en dialectos comarcales. El último baluarte del catalán, la lengua de la burocracia, desaparece con la Guerra de Sucesión y la llegada de los Borbones, con la centralización de las estructuras administrativas que acelera la decadencia. La separación comarcal parece abocar a la lengua a un destino dialectal y, sin embargo, Cataluña reservaba la sorpresa de la Renaixença; en muy poco tiempo se produce un «miracolo letterario».

Cosa avvenne in Catalogna, e concretamente nel suo centro Barcellona? Portando a maturazione il moto di ripresa della tradizione nazionale, già iniziato nell'aspetto formale e linguistico nel secolo precedente da parte di scrittori e poeti come Aribau, Verdaguer, Guimerà, Oller e altri, tra i quali basti ricordare il filologo Milá i Fontanals, un gruppo di personalità eccezionali, guidati da uno dei più acuti critici della civiltà, Eugeni d'Ors, dichiarò inattuale la loro vocazione ideologica, ruppe i legami con l'oligarchia statale e burocratica e, tramite l'adozione di un atteggiamento cosmopolita che li collegava direttamente all'intellettualità progressiva d'oltre Pirenei, riprese con efficacia la bandiera delle lettere in decadenza [...]. La sensazione più immediata è di trovarsi di fronte a un miracolo letterario: una letteratura che aveva avuto il suo secolo d'oro fra il Trecento e il Quattrocento, che aveva fino all'Ottocento subito una decadenza tale da far parlare di scomparsa e di folclorizzazione, o benevolmente di latitanza, offre quasi d'incanto un panorama di attività straordinario, che appena in mezzo secolo avrà fatto coagulare una produzione notevolissima e definito una tradizione. (Grilli 1979: 106.)

La mayor novedad del ensayo analizado radica en la dialéctica que Grilli reconstruye alrededor del Noucentisme, un tema que se había estudiado escasamente en Italia y que instituye un vínculo con el Novecentismo y sus detractores ${ }^{18}$ : «La produzione, soprattutto poetica, che si ispirò o coincise con le posi-

18 «"900", Cahiers d'Italie et d'Europe» fue una revista novecentista, fundada en 1926, dirigida por Massimo Bontempelli y Curzio Malaparte. Tenía como objetivo la internacionalización de la cultura italiana y tuvo como redactores, entre otros, a Ramón Gómez de la Serna, James Joyce, Georg Kaiser, Pierre Mac Orlan. Se publicaron por primera vez unas páginas del Ulysses de Joyce y de Mrs. Dalloway de Virginia Woolf. Después de los primeros cuatro números el régimen fascista impuso el italiano como lengua de publicación y la aventura novecentista terminó en 1929. 
zioni estetiche di Eugeni d'Ors, si battezzò col nome di Noucentisme e subito i suoi rappresentanti entrarono in aperta polemica con l'intellettualità castigliana, soprattutto con il gruppo dei novantottisti» Grilli (1979: 111). En el camino abierto por estos intelectuales, encontramos una mención a Pla:

Nel 1926 intorno alla rivista «L'amic de les Arts», che si pubblicava a Sitges [...] si raccolse un gruppo di artisti scrittori e poeti che si posero fondamentalmente due problemi: quello dei collegamenti internazionali e dei pericoli che la tradizione di poesia colta, erede del Noucentisme, potesse portare ad una chiusura provinciale a causa della sua pretesa autosufficienza. Il pericolo, con prospettiva storica, possiamo individuarlo perfettamente nel caso letterario di un Josep M. Sagarra (1894-1961) e, sia pur con il distanziamento e le specificazioni del caso, nella parabola letteraria, umana e politica di Josep Pla (1897). Pla, eternamente in bilico tra cosmopolitismo e provincialismo deteriore e casolà, rappresenta davvero il punto di congiunzione di una mentalità, di un modo di vivere il catalanismo come vaga avventura culturale e viceversa; si danno infatti in lui per un verso la perseveranza tenace e contadina di un modello linguistico, per l'altro uno scetticismo sincero ma superficiale rispetto ai contenuti culturali. Sta di fatto che Sagarra e Pla, su fronti diversi, impersonano negli anni venti e trenta l'opposizione battagliera al radicalismo delle avanguardie cui talvolta, magari con squisito opportunismo borghese, strizzava l'occhio la cultura ufficiale di filiazione liberal [...]. (Grilli: 1979: 119.)

Vemos como, en el cuadro de una dialéctica entre vanguardias y programas culturales distintos, localistas o cosmopolitas, la figura de Pla se presenta de forma autónoma y con caracteres peculiares. De todas formas, dadas las premisas ideológicas del ensayo, el ampurdanés no parece suscitar las simpatías de Grilli, el cual le dedica más que un juicio estético, un análisis de su postura frente a la situación política, aunque eso sí desde una perspectiva cultural.

La crisis económica de 1929 primero y la Guerra Civil después, le sirven a Grilli como marco para situar la actitud de los escritores e intelectuales catalanes frente a las revoluciones sociales y los procesos históricos de las clases trabajadoras; sus obras son puestas en relación con su postura política y, salvo en casos esporádicos, desde la perspectiva adoptada por el estudioso italiano no se destaca ningún autor relevante: Grilli declara abiertamente que no encuentra a nadie en Cataluña, durante esos trágicos años, a la altura de Antonio Machado.

Con respecto a los oscuros años de la posguerra el ensayo relata la débil supervivencia de los impulsos anteriores al conflicto, la descomposición de la vitalidad cultural de Barcelona, la marginalidad de sus protagonistas, a menudo confinados fuera de las grandes ciudades o dispersos en el exilio. El nombre de Pla, tal vez por inhibición de tipo político, brilla por su ausencia, mientras que la herencia de la mejor creatividad de anteguerra se asocia a la obra de Merce

Los defensores de una Italia tradicional y provincial, reunidos alrededor de la revista «Strapaese», eran sus más directos detractores. 
Rodoreda, una autora que, en efecto, goza en Italia de una duradera y renovada atención.

En el estudio, que llega hasta finales de los setenta, con menciones a autores como Quim Monzó y Carmen Riera, la atención se desplaza a un presente postfranquista, juzgado de forma despiadada:

[...] la dissipazione tematica e formale (...), l'immaginazione al potere, crisi del marxismo, liberazione gay, la scrittura come differenza, gli autori della biblioteca di papà come personaggi, i libri come gioco o pretesto [...]. La prevaricazione propugnata dal contesto sul testo è qui senza attenuanti e senza giustificazioni. Il romanzo freak, in Catalogna ancor più che altrove, stabilisce con la letteratura solo un rapporto di reciproche strumentalizzazioni: volutamente più volgari del puterío delle sue copertine. (Grilli 1979: 162-163.)

El estudio de Grilli no está evidentemente interesado en recuperar a Pla de la marginalidad o irrelevancia a la que su obra parece destinada en su tan querida Italia. El silencio sobre su obra dura hasta los años noventa del siglo pasado, cuando en la isla de Cerdeña se publican dos libros que representan, hasta ahora, lo más relevante del ampurdanés en italiano, en un contexto del todo peculiar y que comentamos a continuación.

\section{LAS TRADUCCIONES DE LOS AÑOS NOVENTA: L'ISOLA DI SAR- DEGNA Y CARA ITALIA}

Publicado en 1991 por la editorial La Celere de Alghero, L'Isola di Sardegna recoge los reportajes y escritos de Pla sobre la isla. El libro se divide en dos partes; en la primera encontramos las memorias de dos viajes del autor a la isla, en 1936 y en 1963, ambas procedentes de Les Illes, según la edición de Destino de 1970; la segunda contiene una la larga entrevista-monólogo al arquitecto sardo Antonio Simon Mossa, recogida en el volumen 29 de la obra completa de Pla, Homenots, cuarta serie, Destino, 1975. En una nota introductoria el editor subraya que la publicación hubiera sido imposible sin la generosidad de Destino «che ha concesso i diritti d'edizione gratificandoci di un trattamento di particolare favore che vuole sottolineare l'opportunità di ampliare i rapporti, anche economici, tra le due comunità che in qualche modo si sentono ancora vicine» (en Pla 1991: VII). La edición de La Celere ha gozado del apoyo de la Regione Sardegna - a través del Assessorato Regionale della Pubblica Istruzione, Beni culturali, Informazione, Spettacolo e Sport- y por su parte, la traducción ha sido posible gracias a la contribución de la Istituciò de les Lletres Catalanes de la Generalitat de Catalunya.

La referida nota introductoria es escueta. En primer lugar se pone de relieve el deseo de aportar a la cultura sarda una visión externa acerca de la historia y la cultura de la isla. Josep Pla, se dice de inmediato, no es un «otro cualquiera», sino un pensador y escritor «fra i maggiori monumenti della letteratura catalana di sempre» (Ivi: X). El libro se inscribe en una colección cuyo propósito apunta a un proceso de recuperación «del patrimonio storico-culturale dell'isola» 
(Ivi: IX) y, podríamos decir, tiene a la propia Cerdeña como objeto principal de investigación.

Quizás por ello, tratándose además de una editorial con escasa repercusión en el resto de Italia, la obra muy presente en las bibliotecas sardas, no se encuentra sin embargo fuera de la isla, tal como pone de manifiesto el catálogo del Opac Sbn, que en Italia presenta una relación de los fondos de la casi totalidad de las bibliotecas de cualquier orden y procedencia. La búsqueda realizada localiza el texto en las instituciones bibliotecarias que se citan a pie de página ${ }^{19}$.

Para la lectura de L'Isola di Sardegna hemos recurrido al ejemplar disponible en la biblioteca de la Universidad Pompeu Fabra. Otros tres ejemplares se encuentran respectivamente en la Biblioteca de Catalunya, en la de la Universitat Autònoma de Barcelona y en la de la Universitat de Vic. La circulación de esta obra parece pues reducida a un espacio sardo-catalán; casi se puede subrayar un eje especial entre la isla y Cataluña, que se ve reforzado por el apoyo que las instituciones correspondientes de las dos autonomías le han otorgado. La presencia, además, de dos importantes departamentos de lengua y cultura catalana en las Universidades de Cagliari y Sassari, es un factor a tener en cuenta al respecto. Sin embargo, este eje sardo-catalán quizás pueda excluir potenciales lectores de Pla entre el resto del público italiano — de hecho imposibilitados de acceder a la obra, por lo menos por lo que se refiere al préstamo bibliotecariopuesto que los escritos traducidos, extrapolados de su contexto -el Mediterráneo y sus islas - parecen situar Cerdeña en un espacio separado, confirmando, paradójicamente, una marginal ubicación cultural.

Notamos además que, al haberse reunido en un único volumen páginas de Pla sobre Cerdeña provenientes de obras diferentes, se repiten varias veces conceptos y hasta frases acerca de un mismo tema. Se acentúa, quizás, un cierto provincianismo y nos atrevemos a sugerir que la obra, así concebida, está destinada a no salir de las bibliotecas de la isla, más allá, desde luego, de la posible negligencia de las demás instituciones bibliotecarias italianas.

Creemos que un exceso de buenas intenciones, por una parte el deseo de los organismos culturales catalanes de dar a conocer la obra de Pla y por otra él de

19 Biblioteca comunale Generale e di Studi Sardi - Cagliari; Biblioteca della Soprintendenza per i beni architettonici e per il paesaggio e per il patrimonio storico, artistico e demoetnoantropologico per le province di Cagliari ed Oristano - Cagliari; Biblioteca del Distretto delle scienze umane - Sezione scienze del linguaggio - Università degli studi di Cagliari - Cagliari; Biblioteca comunale; Quartu Sant'Elena - CA; Biblioteca provinciale Emilio Lussu — Cagliari; Biblioteca regionale - Cagliari; Biblioteca comunale - Sestu - CA; Biblioteca universitaria di Cagliari Cagliari; Biblioteca del Distretto delle scienze umane - Sezione Giordano Bruno - Università degli studi di Cagliari - Cagliari; Biblioteca dell'Istituto di studi occitani - Dronero - CN; Biblioteca comunale - Bosa - OR; Biblioteca del Consorzio per la pubblica lettura Sebastiano Satta - Nuoro; Biblioteca dell'Archivio di Stato di Nuoro - Nuoro; Biblioteca dell'Istituto superiore regionale etnografico - Nuoro; Biblioteca comunale - Oristano; Biblioteca comunale Rafael Sari - Alghero; Biblioteca dell'Archivio di Stato di Sassari - Sassari; Biblioteca Universitaria di Sassari Sassari; Biblioteca della Soprintendenza per i beni archeologici per le province di Sassari e Nuoro — Sassari; Biblioteca della Confraternita della Misericordia - Alghero - SS. 
los organismos culturales sardos de dar a conocer la mirada de un gran escritor catalán sobre la isla, han reducido finalmente esta obra a un diálogo sólo entre dos, algo que quizás ha terminado por limitar su difusión. La hipótesis se refuerza si consideramos que se trata del primer volumen extenso del ampurdanés publicado en Italia, publicación que parece haber trazado un camino de limitado recorrido, lo que se acentúa si se tiene en cuenta la parecida suerte que ha corrido el libro que consideramos a continuación.

Cara Italia es una obra de 1994, publicada por la editorial Edes de Sassari, una ciudad del norte de Cerdeña, y compuesta por escritos de Pla de procedencia distinta. El núcleo principal lo compone Cartes d'Itália, según la edición de 1982 de Destino incluida en Les escales de Llevant, al cual se añaden los siguientes capítulos en un post scriptum:

- Superficie di Roma (pp. 225-234), traducción de Superficie de Roma, en Itália i el Mediterrani, Barcelona, Destino, 1988.

- Sassari, Alghero, Porto Torres, Cagliari (pp. 235-242), en L'isola di Sardegna, Alghero, La Celere, 1991.

- Palermo (pp. 243-247), Palerm, en Le illes, Barcelona, Destino, 1981.

El editor explica, en ese post scriptum, que, en 1937, Pla se encontraba en Roma junto con otros compatriotas, debido a la Guerra Civil Española. Con ellos se reunía en el Caffè Greco de via Condotti, donde conoció a algunos jóvenes sardos; uno de ellos, Antonio Simon Mossa, se expresaba en catalán y le habló de la presencia en Alghero de una colonia de catalano-hablantes, lo que despertó su curiosidad. A raíz de este descubrimiento Pla emprendió su primer viaje a la isla de Cerdeña; durante la estancia en Cagliari, padeció un contratiempo que desencadenó otro viaje: como consecuencia del robo de su cartera en el hotel, el escritor tuvo que desplazarse a Sicilia y de allí emprendió un inesperado recorrido por el Mediterráneo que dio lugar posteriormente a Les illes. Los capítulos que de este libro están dedicados a Italia completan así la edición sarda de Cartes d'Itália. En el caso de Sassari, Alghero, Porto Torres y Cagliari se trata de un préstamo del volumen L'isola di Sardegna, mientras que en los casos restantes nos encontramos con traducciones hasta aquel momento inéditas, llevadas a cabo con el sostén de la «Institució de le Letres Catalanes» de la Generalitat de Catalunya.

En el documentado prólogo de Cara Italia, firmado por Ignazio Delogu, docente de la Università degli Studi di Sassari, se presenta a Pla teniendo en cuenta su escaso conocimiento entre el público italiano. Desde las primeras líneas nos topamos con una característica común a las presentaciones del ampurdanés en Italia. Se trata del reconocimiento de su extraordinaria importancia, la cual contrasta con su escasa fortuna editorial en el Bel Paese: «Fra gli scrittori europei che hanno frequentato l'Italia fra le due guerre mondiali, Josep Pla è sicuramente quello che ne ha scritto più diffusamente, con conoscenza non superficiale e con simpatia apertamente dichiarata» (Delogu, en Pla 1994: 5). 
El prólogo sigue con un repaso a la dilatada aventura italiana de Pla, aventura que empieza en Génova en 1922, como corresponsal del diario «La Publicidad» de Barcelona, dentro de una más amplia actividad como periodista ejercida a través de distintos lugares del continente europeo, durante más de cinco años. Delogu pone el acento en sus agudas observaciones sobre varios aspectos del país - la lengua y los dialectos, la situación económica, la vida cotidiana del pueblo, el arte, el urbanismo, la industrialización, una pobreza todavía muy común, los contrastes, y un largo etc.- y en como la historia entra de lleno en sus escritos por causa de algunas circunstancias excepcionales que se dan durante sus estancias en Italia. Es el caso de la toma del poder por parte del fascismo, con la marcha sobre Roma de 1922, que sorprende a Pla en un tren rumbo a la capital, adonde se dirigía desde Florencia y del que los camisas negras le obligan a descender junto con los demás pasajeros. Su inicial visión del fascismo, subraya Delogu, fue inicialmente, «come per altri stranieri», de simpatía e interés:

Nazionalismo, spirito antiborghese, rifiuto della democrazia non potevano non risultare congeniali a un giovane che aveva nel suo bagaglio culturale e politico una dichiarata inclinazione verso la destra tradizionalista e nazionalista, compresa l'Action française di Ch. Maurras, dal cui programma politico prenderà successivamente le distanze, ma i cui intellettuali continuerà a considerare come suoi maestri. Anche la sua breve militanza politica in Catalogna era stata nelle file di un movimento nazionalista conservatore come la Lliga nacionalista di F. Cambó [...]. (Delogu, en Pla 1994: 6.)

La cuestión política entra así de lleno en el retrato del ampurdanés, del cual se subraya su visión crítica con respecto a la democracia, que determinó aquella visión del fascismo como «[...] «la manifestazione più raffinatamente realista e conservatrice che è capace di esprimere il capitalismo nazionalista»» (Ivi: 7). Sería incorrecto, de todas formas, imaginar la exclusión de Pla del canon de los grandes escritores europeos en Italia a raíz de su posición política de aquellos años, máxime teniendo en cuenta la adhesión al fascismo, temprana ${ }^{20}$ o definitiva, de gran parte de los propios intelectuales italianos, entre los cuales, solo por citar a algunos, están Gabriele D’Annunzio, Luigi Pirandello o Curzio Malaparte ${ }^{21}$.

${ }^{20}$ El distanciamiento y la desilusión de Pla con respecto al fascismo y a Mussolini evolucionan rápidamente hacia el repudio, coincidiendo en ello con lo que sucede en el caso de muchos intelectuales italianos de la época, exceptuando a los más comprometidos y fieles. La violencia del régimen, su ambigüedad con respecto a la lucha de clases y sindical, sus alianzas oportunistas, la insoportable retórica mussoliniana, el patriotismo beligerante, despiertan en él la nostalgia de la conciencia liberal de la Europa de la anteguerra.

${ }^{21}$ En 1925 Giovanni Gentile escribió el «Manifesto degli intellettuali fascisti», firmado por casi 250 intelectuales, reunidos en un congreso en Bolonia y que posteriormente se publicó en distintos diarios el 21 de abril. Además de Pirandello, se recuerda el apoyo de Filippo Tommaso Marinetti y otros futuristas. La idea de Gentile, acerca de la relación entre el estado y el individuo, preveía una superposición entre ambas partes, puesto que, según el máximo teórico del fascismo, el estado representa la síntesis de las voluntades individuales. Como consecuencia, no hay distin- 
Concluido el excurso inevitable sobre el tema «Pla y el fascismo», que, por otro lado representa una página acorde con el macro tema «intelectuales y fascismo» (Bobbio 1973: passim), la atención de Delogu se ocupa del vivaz cosmopolitismo del escritor, quién, a través de sus viajes a Inglaterra, a la Unión Soviética y a su amada Francia, afina su cultura, amplía su visión del mundo y desarrolla un personal estilo de periodismo cultural, que alcanza a menudo la profundidad del ensayo breve. Desde el punto de vista estilístico, según el estudioso, la escritura de Pla mantiene fuertes lazos con la de otros pensadores y memorialistas de la época, como fueron Guido Gobetti y Antonio Gramsci en Italia o el peruano J. C. Mariátegui. Se trata de un análisis comparado que todavía no se ha llevado a cabo y que, de hacerse, podría dar resultados interesantes.

$\mathrm{Si}$ bien el aspecto estrictamente literario y estilístico de la obra de Pla es analizado por Delogu, que destaca su refinamiento, el aspecto político vuelve una y otra vez, incluso dentro del breve espacio del prólogo que acompaña Cara Italia. La figura de Pla parece mancharse a raíz de su desconfianza hacia la II República, desarrollada durante su larga estancia en Madrid, y que ya en su época suscitó, como es sabido, múltiples antipatías tanto en Cataluña como en el resto de España. Delogu recoge ampliamente esa desafección por la República, que se hizo más explícita con su vuelta a Barcelona detrás de las tropas franquistas y con su nombramiento como director del diario «La Vanguardia».

Aunque el estudioso sardo no se pronuncia al respecto, tal vez el posicionamiento de Pla con respecto a la Guerra Civil española haya proyectado una sombra sobre su biografía, que puede haber interferido, por lo que se refiere a su circulación en Italia, más que su temprano entusiasmo por Mussolini.

El hecho indudable es que durante más de cincuenta años ninguna obra de Pla fue traducida al italiano; el rescate del olvido se da, además, en circunstancias peculiares y dentro de un área del país, la de Cerdeña, todo lo cual parece confinarlo a una recepción local y condicionada. Y esto a pesar de las intenciones de quienes, con evidente empeño y dedicación, han subrayado el extraordinario legado del ampurdanés en sus escritos sobre Italia:

Non c'è, infatti, viaggiatore e scrittore che abbia respinto e confutato con maggior forza, lucidità e passione la somma di luoghi comuni e correnti accu-

ción entre privado y público y no hay posibilidad de limitar la acción del estado. Se trata evidentemente de una visión antiliberal y antidemocrática que parece encontrar un primer apoyo por parte de Pla. Sin embargo, en el reportaje dedicado a la ciudad de Bari encontramos una referencia que situa claramente al ampurdanés en un territorio ideológico bien distinto: «[...] dopo una visita alla librería editoriale Laterza, editore di «La Critica» di Croce e di quasi tutti i libri di don Benedetto e dei suoi amici - il gruppo di intellettuali più rispettabile della penisola di questa prima metà del secolo» (Pla 1994: 142). La referencia a Benedetto Croce y el juicio que atribuye a su círculo, definido como el más respectable grupo intelectual de la península, llama en causa el «Manifesto degli intellettuali antifascisti», publicado en la revista «Il Mondo» el $1^{\circ}$ de mayo de 1925 , en calidad de «risposta di scrittori, professori e pubblicisti italiani al manifesto degli intellettuali fascisti. Entre los firmantes estaban Luigi Einaudi, Giovanni Amendola, Matilde Serao, Eugenio Montale, Aldo Palazzeschi y Mario Casella. Gracias al prestigio del que gozaba Croce, dentro y fuera de Italia, el régimen toleró su oposición, que postulaba la autonomía de la cultura frente a la política. 
mulatisi nel tempo sull'Italia e sugli italiani. [...] Nessuno che abbia colto con pari acutezza e liberalità gli elementi che caratterizzano i comportamenti di un popolo troppe volte accusato con superficialità e forse non senza tendenziosità di difetti comuni a ogni altro popolo europeo. (Ivi: 13.)

Al hablar de una especie de confinamiento de Pla a sectores muy reducidos de la cultura italiana ${ }^{22}$, y en particular a los especialistas en el ámbito de la cultura catalana de las universidades de Cerdeña, no queremos de ninguna manera disminuir la labor de traducción y edición llevada a cabo, siendo por otro lado el volumen en cuestión, Cara Italia, una excelente prueba de ello. Es indudable, sin embargo, la escasa circulación de la obra del autor y al respecto es suficiente comprobar la difusión del volumen en las bibliotecas del país. Utilizando de nuevo para la búsqueda el catálogo del Opac Sbn, constatamos que Cara Italia sólo se encuentra disponible en las cinco instituciones bibliotecarias que se indican a pie de página, todas ellas de Cerdeña ${ }^{23}$.

Si a estas instituciones sumamos los ejemplares encontrados en Cataluña -Biblioteca de Catalunya, Universitat Autònoma de Barcelona, Universitat de Girona-Biblioteca Josep Pla, Palafrugell y Universitat Pompeu Fabra - vemos por segunda vez como la recepción del ampurdanés se limita al eje sardo-catalan. Y esto ocurre, por añadidura, con un texto tan propicio, para suscitar el interés entre los lectores italianos, como es Cara Italia.

\section{CONCLUSIONES}

Hemos visto que a Josep Pla se le reconoce en Italia como uno de los más destacados escritores catalanes del siglo XX, mientras que su obra no goza de una difusión a la altura de esa valoración. Este hecho no está vinculado sin embargo ni a una inadecuada ubicación de las letras catalanas en la cultura italiana, ni a una escasa difusión de sus escritores. Al respecto, es conveniente referirse, aunque sea brevemente, a la circulación de la obra de otros autores catalanes en el Bel Paese, como son por ejemplo otros dos tótems del siglo XX, Merce Rodoreda y Salvador Espriu.

22 Señalamos, para completar la reseña de los escritos del ampurdanés traducidos al italiano, Itineraio per Napoli/Itinerari pèr Napols, un texto en edición bilingüe publicado en 2005 por el Institut Ramon Llull en ocasión de la semana de la cultura catalana que se celebró del 7 al 15 de octubre del mismo año en la capital de Campania. En la introducción, a cargo de Rossend Arqués, se habla de los viajes de Pla a Nápoles y de como sus distintos escritos sobre la ciudad han ido evolucionando hasta los realizados para Cartes d'Itália. El texto recopila, a la manera de un itinerario turístico-cultural, algunas de las páginas más sugerentes del escritor alrededor de núcleos importantes de la ciudad (el Teatro Bellini, la Cappella San Severo in Santa Maria della Poeta, Spaccanapoli, Via Toledo, etc.).

${ }^{23}$ Biblioteca regionale - Cagliari; Biblioteca universitaria di Cagliari; Biblioteca comunale Rafael Sari - Alghero; Biblioteca del Conservatorio di musica Luigi Canepa - Sassari; Biblioteca di Sardegna - Cargeghe - SS. 
La obra más conocida de Rodoreda, La Plaça del diamant, se ha editado en Italia en tres ocasiones, entre 1970 y 2008 , cada una con una nueva traducción. La última, de 2008, publicada por La nuova frontiera, ha representado un verdadero acontecimiento literario inesperado incluso para sus propios editores. La atención de varios medios de comunicación — diarios, suplementos literarios, programas radiofónicos y hasta un célebre programa televisivo- - han convertido la historia de Colometa, traducida ahora por primera vez al italiano como Colombetta, en un best seller que ha superado con creces las dos anteriores ediciones. Según un estudio de Annamaria Annichiarico, La Piazza del Diamante, además de haber vendido 40.000 ejemplares sólo en 2008, fue elegida en 2009 como libro del año por las preferencias del público de Fahrenheit, el programa radiofónico dedicado a la literatura de la Radio Rai $3^{24}$. Otras obras de la escritora cuentan con ediciones muy elogiadas, como es el caso de Lo specchio rotto (1992) Quanta, quanta guerra (1994), Viaggi e fiori (1995), las tres editadas por la prestigiosa Bollati Boringhieri de Turín, Via delle camelie (1991) por el editor La tartaruga de Milán, y hasta una traducción al sardo, Sa pratza de su diamante (2008), por el editor Papiros de Nuoro.

Al igual que de Rodoreda, de Salvador Espriu se han traducido al italiano varias obras y desde luego las más representativas. Es el caso del volumen que reúne Pelle di toro; Libro di Sinera; Le canzoni di Arianna (1966), por el importante editor Guanda de Parma, que cuenta con una introducción, traducción y notas de Adele Faccio $^{25}$ y un post scriptum del poeta Alfonso Gatto. En 1989 ortos poemas de Espriu se dan a conocer con Cristallo di parole, por el editor Japani de L'Aquila, en la colección «Poeti e prosatori catalani»; de su obra teatral se ha traducido al italiano Antigone, en tres sucesivas ediciones (Palermo, Ars, 1988; Firenze, Aleteia, 1996; Roma, Bonanno, 2010) y Fedra (Roma, Bonanno, 2011).

Así pues, el hecho de la escasa circulación de la obra de Pla se pone más en evidencia al compararlo con la distinta suerte corrida por la obra de Rodoreda y de Espriu, y más aún si se tiene en cuenta que desde los años noventa asistimos a un fuerte impulso editorial de la literatura catalana en Italia (Mongiardo 2012:

${ }^{24}$ Explica Annichiarico que el éxito de La Piazza del Diamante, además de ser el resultado de una dosis del insondable azar del destino, ha de ser interpretado teniendo en cuenta el renovado interés hacia la cultura catalana en Italia, determinado en parte por el amplio tratamiento que el mundo académico le ha reservado en tiempos más recientes, pero también al éxito en sí de la propia Cataluña, propiciado por unas relaciones cada vez más estrechas entre los italianos y Barcelona, en primer lugar. Un sistema compuesto por la gastronomía, el teatro, el cine, la música, el turismo, etc., ha situado Cataluña en un mapa privilegiado del público italiano, factor que conlleva una mayor visibilidad de sus autores. No acaso, en 2002, Cataluña es el país invitado de la Fiera del libro di Torino, la más importante de Italia, que anticipa de esta manera la Buchmesse de Frankfurt en 2007.

${ }^{25}$ Adele Faccio (1920-2007) ha sido una destacada política y diputada italiana del Partito Radicale. Sobrina de la escritora Sibilla Aleramo, cuyo verdadero nombre era Marta Felicina Faccio, antes de darse a conocer por su compromiso a favor de una ley de plazos para el aborto, fue profesora de idiomas y traductora para la editorial Mondadori de Milán. Residió durante un tiempo en Barcelona, donde aprendió catalán y entró en contacto con Salvador Espriu. 
passim), que culmina, en 2002, con la presencia de Cataluña como país invitado de la «Fiera del libro di Torino» ${ }^{26}$.

A la luz de lo visto hasta ahora se resumen las dos principales circunstancias que pueden haber influido en la escasa fortuna, en cuanto a la difusión de su obra, del escritor ampurdanés en Italia:

Razones políticas. El juvenil entusiasmo fascista, creemos, no ha representado un obstáculo a su circulación, sobre todo si se tiene en cuenta su rápido repudio y el clima general de la época en los años del ascenso de Mussolini. Un razonamiento distinto se puede hacer con respecto a su postura durante y después de la Guerra Civil. A pesar de su distanciamiento personal con el régimen franquista, que por otro lado no se dio en términos de oposición manifiesta, una mancha política puede haber influido en el escaso interés hacia su obra, junto con la dificultad intrínseca de su escritura y el género espurio de sus crónicas, las cuales presentan una mayor complejidad de lectura que la obra de aquellos escritores que eligen el camino de la novela. Su ausencia se explicaría así sobre todo en relación con la renovada atención en Italia hacia Cataluña en los años setenta del siglo XX, la cual se enmarca en una crítica literaria militante de izquierda; el pasado político del autor, atendiendo a su posición en las décadas inmediatas al final de la Guerra Civil española, puede haber jugado, por lo menos en ese momento, en contra de su recepción;

Confinamiento insular. Las traducciones llevadas a cabo por editores de Cerdeña en los años noventa, con intervención de la Generalitat de Catalunya dentro de su «reivindicación» de Alghero como parte del área lingüística catalana, pueden haber paradójicamente determinado una provincialización del autor, percibido como un representante de un eje cultural ajeno y poco interesante para el resto de Italia. Cierto es que las dos principales traducciones de Pla al italiano se encuentran disponibles solo y exclusivamente en bibliotecas de Cerdeña y de Cataluña vinculadas a entornos académicos. Puede tratarse de una cuestión merecedora de más matices de los aquí expuestos aunque se aprecian como complementarios a la línea desarrollada en el presente artículo y que, con seguridad, serán merecedores de un futuro desarrollo.

Añadimos, a modo de conclusión, otras circunstancias que pueden haber influido en la ausencia de otras obras significativas de Pla en Italia. Sorprende, teniendo en cuenta además que el Quadern gris ha sido publicado en Francia por Gallimard, el hecho de que ninguna editorial italiana haya incluido esta obra en su catálogo, ni siquiera en 2002, en ocasión de la Fiera del libro di Torino. Puede haber pesado, en este sentido, una difidencia con el género de los dietarios, que, en efecto, en Italia no tiene la misma gran tradición que en Francia. Ciertas peculiaridades de la obra mayor de nuestro escritor, además, como son su extensión, su estilo, las referencias a hechos y personalidades de Cataluña poco inteligibles

26 La Fiera internazionale del libro di Torino es el más importante encuentro editorial en Italia, abierto tanto a los profesionales del sector, como al público de los lectores. Por número de editoriales que participan en ella es la segunda feria en Europa, después de la Buchmesse de Frankfurt, y la primera en cuanto a número de visitantes. 
sin un adecuado apartado crítico, pueden haber contribuido a rebajar el interés de los editores. Es posible que Pla se considere un autor difícil de traducir y de pre$\operatorname{sentar}^{27}$. Es preciso, al respecto, mencionar un último dato. En los programas de la asignatura de Lengua y Literatura catalana que se imparten en numerosas universidades italianas, la obra de Pla está casi siempre incluida. Se considera, tal vez por ello, que el público interesado al autor puede leer su obra directamente en catalán y, desde la perspectiva comercial, esto explique su ausencia de los catálogos de las más importantes editoriales italianas.

Todo parece indicar, en definitiva, que el hecho catalán, por así llamarlo, como ámbito lingüístico, literario y cultural, queda en Italia perfectamente reconocido y asimilado, a pesar de algunas discontinuidades. Se ha de excluir cualquier miopía al respecto, vista la sólida presencia de un tejido de estudiosos, traductores y conocedores de la literatura catalana en Italia: el éxito fenomenal, como diría el propio Pla, de la obra de Rodoreda, no deja margen para dudas. La escasa presencia del ampurdanés es, a todos los efectos, un caso peculiar.

\section{BIBLIOGRAFÍA}

Annichiarico, A. (2011) «Da Colometa a Colombetta: ancora una traduzione de "La plaça del Diamant" di Mercè Rodoreda», Rivista italiana di studi catalani, 1, pp. 91-10.

Arqués, R. (2002) «Josep Pla: Itàlia com a mirall», Quaderns d'Italià, 7, pp. 187-206.

Bignamini, I. (1997): Grand Tour: il fascino dell'Italia nel XVIII secolo, Milano, Skira.

Bobbio, N. (1973) La cultura e il fascismo, en Fascismo e società italiana, a cura di Guido Quazza, Torino, Einaudi, pp. 209-246.

Cardona, E. (1878) Dell'antica letteratura catalana: studii di Enrico Cardona; seguiti dal testo e dalla traduzione della vita di Giacomo 1. tolta dalla Cronaca catalana di Ramon Muntaner, Napoli, Gargiulo.

Casella, M. (1918) Agli albori del Romanticismo e del moderno Rinascimento catalano, «Rivista delle biblioteche e degli archivi», XXIX, Firenze,

${ }^{27}$ Quien escribe ha comentado la cuestión que nos ocupa en conversaciones informales con algunos editores italianos, los cuales concuerdan básicamente en lo siguiente: la obra de Pla, que en absoluto se desconoce entre quienes se ocupan de los autores de la península ibérica, presenta múltiples dificultades. En primer lugar, las memorias, observaciones, reflexiones y apuntes de viajes de un casi desconocido - como es Pla en Italia - difícilmente, se cree, podría despertar el interés del público. En segundo lugar, la continua referencia a una gran cantidad de nombres y apellidos de personajes prácticamente desconocidos en Italia, complica el asunto, al no ser que la potencial edición se dote de un importante apartado de notas. En tercer lugar la escritura del ampurdanés se considera difícil y, debido además a su gran extensión, presupone una traducción compleja, larga y, en fin, cara. 
pp. 81-120 [Id. (1966), en Saggi di letteratura provenzale e catalana, Bari, Adriatica Editrice, pp. 307-371].

De Seta C. (1992) L'Italia del grand tour: da Montaigne a Goethe, Napoli, Electa.

Grilli, G. (1979) La letteratura catalana: la diversità culturale nella Spagna moderna, Napoli, Guida.

Macrí, O. (1976) Varia fortuna del Manzoni in terre iberiche, Ravenna, Longo.

Mongiardo, L. (2012) «Omaggio alla Catalogna», Rivista tradurre, 2, Torino, http://rivistatradurre.it/2012/05/omaggio-alla-catalogna/.

Pagano, J. L. (1902) Attraverso la Spagna letteraria. I Catalani: colloqui con Angel Guimerà, Pompeyo Gener, Joan Maragall, Roma, Ed. della Rassegna Internazionale.

- (1964) Evocaciones. Ensayos, Buenos Aires, Academia Argentina de Letras.

Pla, J. (1926) Pietro Brincs uomo di Bagur, en Ravegnani, cit., pp. 230-236.

- (1983) Cartes d'Itália, Barcelona, Destino.

- (1983) Quadern gris, Vol. I-II, Barcelona, Destino.

- (1991) L'isola di Sardegna, traduzione di Antoni Arca, Alghero, La Celere.

- (1994) Cara Italia. Lettere e cartoline di un tempo che fu, traduzione a cura di Antoni Arca, prologo di Ignazio Delogu, Sassari, Edes.

- (2005) Itinerario per Napoli, a cura di Rossend Arqués, Barcelona, Institut Ramon Llull.

Raimondi, E. (1997) Letteratura e identità nazionale, Milano, Bruno Mondadori.

- (1997) Romanticismo italiano e romanticismo europeo, Milano, Bruno Mondadori.

Ravegnani, G. (1926) Antologia di novelle catalane, Milano, Firme nuove.

Rigobon, P. (2012) «Recenti traduzioni italiane di narrativa catalana», Rivista di studi catalani, 2, pp. 157-172.

Rodoreda, M. (2008) La Piazza del Diamante, traduzione e postfazione di G. Tavani, nota di lettura di Sandra Cisneros, Roma, La Nuova Frontiera. 